\title{
On the first place antitonicity in QL-implications
}

\author{
Y. Shi \\ Ghent University, \\ Krijgslaan 281 (S9), \\ 9000 Gent, Belgium \\ Yun.Shi@UGent.be
}

\author{
D. Ruan \\ Belgian Nuclear Research Centre \\ $(\mathrm{SCK} \bullet \mathrm{CEN}), 2400 \mathrm{Mol}$, \& \\ Ghent University, \\ Krijgslaan 281 (S9), \\ 9000 Gent, Belgium \\ druan@SCKCEN.BE
}

\author{
E.E. Kerre \\ Ghent University, \\ Krijgslaan 281 (S9), \\ 9000 Gent, Belgium \\ Etienne.Kerre@UGent.be
}

\begin{abstract}
In order to obtain a demanded fuzzy implication, a number of properties have been proposed, among which the first place antitonicity, the second place monotonicity and the boundary conditions are the most important ones. The three classes of fuzzy implications derived from the implication in binary logic, S-, R- and QL-implications all satisfy the second place monotonicity and the boundary conditions. However, not all the QL-implications satisfy the first place antitonicity as S- and R-implications do. In this paper we study the QL-implications satisfying the first place antitonicity. First we study the relationship between the first place antitonicity and other required properties of QLimplications. And then we work on the conditions under which a QL-implication generated by different combinations of a t-conorm $S$, a t-norm $T$ and a strong fuzzy negation $n$ will satisfy the first place antitonicity, especially on the cases that both $S$ and $T$ are continuous. We also investigate the interrelationships between $\mathrm{S}$ - and R-implications on one hand and QL-implications satisfying the first place antitonicity on the other.
\end{abstract}

Keywords: Fuzzy implication, QLimplication, the First place antitonicity.

\section{Introduction}

A fuzzy implication is a fuzzy connective that has played important roles in different fuzzy domains $[5,6,7,13,14]$. There are several different definitions of a fuzzy implication, e.g., [1, 3, 4, 9]. In this paper we define a fuzzy implication as a $[0,1]^{2} \rightarrow[0,1]$ mapping that satisfies the boundary conditions:

I0. $\mathrm{I}(0,0)=\mathrm{I}(0,1)=\mathrm{I}(1,1)=1, \mathrm{I}(1,0)=0$
One of the fuzzy inference methods is the generalized modus ponens. In fuzzy logic, the generalized modus ponens is realized through IF-THEN rules. Let $X$ and $Y$ be two linguistic variables on the universe of discourses $U$ and $V$ respectively. Moreover, let $A$ and $A^{\prime}$ be two fuzzy sets on $U$ and let $B$ and $B^{\prime}$ be two fuzzy sets on $V$. $A, A^{\prime}, B$ and $B^{\prime}$ may refer to linguistic concepts. An IF-THEN rule is expressed by:

IF $X$ is A, THEN $Y$ is B $X$ is $A^{\prime}$

$\mathrm{Y}$ is $B^{\prime}$

where $B^{\prime}$ is obtained through Zadeh's compositional rule of inference:

$$
B^{\prime}(v)=\sup _{u \in U} T\left(A^{\prime}(u), I(A(u), B(v))\right)
$$

In this formula, $T$ is a t-norm and $I$ is a fuzzy implication. In order to obtain a suitable conclusion of the fuzzy inference, a number of properties have been proposed for the fuzzy implication $I[4,10,11,15,17]$, among which the most important ones are:

I1. the first place antitonicity: $x_{1}<x_{2} \Rightarrow I\left(x_{1}, y\right) \geq I\left(x_{2}, y\right)$, for all $x_{1}, x_{2}, y \in[0,1]$;

I2. the second place monotonicity: $y_{1}<y_{2} \Rightarrow I\left(x, y_{1}\right) \leq I\left(x, y_{2}\right)$, for all $x, y_{1}, y_{2} \in[0,1]$;

I3. the neutrality property: $I(1, x)=x$, for all $x \in[0,1]$;

I4. the exchange principle: $I(x, I(y, z))=I(y, I(x, z))$, for all $x, y, z \in[0,1] ;$

I5. the ordering property: $x \leq y \Leftrightarrow I(x, y)=1$, for all $x, y \in[0,1] ;$

I6. the contrapositive principle: $I(x, y)=I(n(y), n(x))$, for all $x, y \in[0,1]$, w.r.t. a strong fuzzy negation $n$; 
I7. the continuity.

I1 and $\mathrm{I} 2$ are the most important properties. Some authors even define a fuzzy implication $I$ as a $[0,1]^{2} \rightarrow$ $[0,1]$ mapping that satisfies I1, I2 and

I9 $I(0, x)=1$, for all $x \in[0,1]$;

I10 $I(x, 1)=1$, for all $x \in[0,1]$;

I11 $I(1,0)=0$.

Notice that if $I$ satisfies I0, I1 and I2, then I9, I10 and I11 will be satisfied immediately.

There are three important classes of fuzzy implications derived from the implication in binary logic ([11], Chapter 11):

1. Strong implication (S-implication), $I(x, y)=S(n(x), y)$, where $S$ is a t-conorm and $n$ is a strong fuzzy negation;

2. Residuated implication (R-implication), $I(x, y)=\sup \{t \in[0,1] \mid T(x, t) \leq y\}$, where $T$ is a t-norm;

3. Quantum logic implication (QL-implication), $I(x, y)=S(n(x), T(x, y))$, where $S$ is a t-conorm, $T$ is a t-norm and $n$ is a strong fuzzy negation.

All these three classes of fuzzy implications satisfy I0 and I2. However, although all S-implications and Rimplications satisfy I1 ([10], Definition 1.15), not all the QL-implications do.

Some work on whether a QL-implication satisfies I1 or not has been done in [8], [16] and [12]. In [8], the conditions under which a QL-implication $I_{Q L}$ and a t-norm $T_{*}$ satisfy the residuation property: $T_{*}(x, z) \leq y \Leftrightarrow$ $z \leq I_{Q L}(x, y)$, for all $x, y, z \in[0,1]$ are found. This means that $I_{Q L}$ is an R-implication as well ([8], Example 4.5). Hence $I_{Q L}$ satisfies I1 provided these conditions are fulfilled. However, being an R-implication is sufficient but not necessary for a QL-implication to satisfy I1 (see Remark 4, 5, 6 in this paper). In [16], the authors have worked out how a QL-implication satisfies I1 as well as I4 ([16], Definition 1, Theorem 7, Theorem 11). It is proved that such a QL-implication is an S-implication as well. Again, being an S-implication is sufficient but not necessary for a QL-implication to satisfy I1 (see Remark 5, 6 in this paper). And in [12], the authors have worked out for a group of QL-implications the conditions under which they satisfy I1. They restrict the relationship between the tconorm and the strong fuzzy negation which construct the QL-implications ([12], Proposition 9).

In this paper, we study the QL-implications generated by a t-conorm $S$, a t-norm $T$ and a strong fuzzy negation $n$ that satisfy I1, especially for the cases that both $S$ and $T$ are continuous. First the relationship between I1 and the other properties of QL-implications is studied in Section 3.1. And then the conditions under which a QL-implication satisfies I1 are obtained in Section 3.2. Some QL-implications that satisfy I1 are equivalent to $\mathrm{S}$-implications or R-implications while others are not. We denote these facts in Remark 4 to 7. Preliminaries are given in Section 2 and conclusions are given in Section 4 respectively.

\section{Preliminaries}

Definition 1. An automorphism of the interval $[a, b] \subset \boldsymbol{R}$ is a continuous, strictly increasing mapping $\varphi$ from $[a, b]$ to $[a, b]$ with boundary conditions $\varphi(a)=a$ and $\varphi(b)=b$ ([4], Definition 0).

Lemma 1. If $\phi$ is an automorphism of the unit interval, then $\phi^{-1}$ is also an automorphism of the unit interval.

Lemma 2 (The chain rule). The composition of two automorphisms of the unit interval is again an automorphism.

Definition 2. Two mappings $F, G:[0,1]^{n} \rightarrow[0,1]$ are conjugated to each other, if there exists an automorphism $\phi$ of the unit interval such that $G=F_{\phi}$, where $F_{\phi}\left(x_{1}, x_{2}, \cdots, x_{n}\right)$ $=\phi^{-1}\left(F\left(\phi\left(x_{1}\right), \phi\left(x_{2}\right), \cdots, \phi\left(x_{n}\right)\right)\right)$, $x_{1}, x_{2}, \cdots, x_{n} \in[0,1]$ ([2], Definition 2).

It is easy to see that $G=F_{\phi} \Leftrightarrow F=G_{\phi^{-1}}$.

Definition 3. A mapping $n:[0,1] \rightarrow[0,1]$ is a fuzzy negation if it is decreasing and satisfies: $n(0)=1$, $n(1)=0$.

Definition 4. A fuzzy negation that satisfies $n(n(x))=x$, for all $x \in[0,1]$ is called a strong fuzzy negation.

We denote the standard strong fuzzy negation as $n_{0}$, i.e., $n_{0}(x)=1-x$, for all $x \in[0,1]$.

Definition 5. Let $\phi$ be an automorphism of the unit interval. Then $n_{\phi}$ denotes the strong fuzzy negation that is conjugated to $n_{0}$, i.e., $n_{\phi}(x)=\phi^{-1}(1-\phi(x))$, for all $x \in[0,1]$.

Definition 6. A mapping $T:[0,1]^{2} \rightarrow[0,1]$ is a triangular norm (t-norm for short) if for all $x, y, z \in[0,1]$ it satisfies:

T1. boundary condition: $T(x, 1)=x$;

T2. monotonicity: $y \leq z \Rightarrow T(x, y) \leq T(x, z)$;

T3. commutativity: $T(x, y)=T(y, x)$; 
T4. associativity: $T(x, T(y, z))=T(T(x, y), z)$.

Three important continuous t-norms are:

1. $T_{M}(x, y)=\min (x, y), \quad($ minimum $)$

2. $T_{P}(x, y)=x y, \quad$ (product)

3. $T_{\mathrm{E}}(x, y)=\max (x+y-1,0)$, (bounded product)

Definition 7. Let $\phi$ be an automorphism of the unit interval and $T$ be a t-norm. Then $T_{\phi}$ denotes the $t$ norm that is conjugated to $T$, i.e.,

$T_{\phi}(x, y)=\phi^{-1}(T(\phi(x), \phi(y)))$, for all

$(x, y) \in[0,1]^{2}$.

$T_{M}$ is conjugated to itself, i.e., if $\phi$ is an automorphism of the unit interval, then $T_{M}=T_{M \phi}$.

Definition 8. Let $\left\{\left[a_{m}, b_{m}\right]\right\}$ be a non-empty family of non-overlapping, closed, proper subintervals of $[0,1]$ and $\left\{\phi_{m}\right\}$ be a family of automorphisms of the unit interval. Then a continuous t-norm $T_{o}$ is called an ordinal sum of $\left\{\left[a_{m}, b_{m}\right], T_{m}\right\}$, where $T_{m}=T_{P \phi m}$ or $T_{m}=T_{E \phi m}$, if $T_{o}(x, y)$ is equal to:

$\left\{\begin{array}{l}a_{m}+\left(b_{m}-a_{m}\right) T_{m}\left(\frac{x-a_{m}}{b_{m}-a_{m}}, \frac{y-a_{m}}{b_{m}-a_{m}}\right),(x, y) \in\left[a_{m}, b_{m}\right]^{2} \\ T_{M}(x, y), \quad \text { otherwise }\end{array}\right.$

If there exists only one subinterval $\left[a_{1}, b_{1}\right]$ of $[0,1]$ with $a_{1}=0, b_{1}=1$ and $\phi_{1}$ being the automorphism of the unit interval, then $T_{o}=T_{P \phi 1}$ or $T_{o}=T_{\mathrm{\ell} \phi 1}$. In this paper, as to 'an ordinal sum of $\left\{\left[a_{m}, b_{m}\right], T_{m}\right\}$ ', we mean that there exists at least one subinterval $\left[a_{k}, b_{k}\right]$ such that $a_{k} \neq 0$ or $b_{k} \neq 1$.

It is stated in ([10], Section 1.3.4) that a continuous t-norm is either $T_{M}$, or conjugated to $T_{P}$, or conjugated to $T_{\mathrm{E}}$, or an ordinal sum of the non-empty family $\left\{\left[a_{m}, b_{m}\right], T_{m}\right\}$ with $T_{m}$ being conjugated to $T_{P}$ or $T_{\mathrm{E}}$.

Definition 9. A mapping $S:[0,1]^{2} \rightarrow[0,1]$ is a triangular conorm (t-conorm for short) if for all $x, y, z \in[0,1]$ it satisfies:

S1. boundary condition: $S(x, 0)=x$;

S2. monotonicity: $y \leq z \Rightarrow S(x, y) \leq S(x, z)$;

S3. commutativity: $S(x, y)=S(y, x)$;

S4. associativity: $S(x, S(y, z))=S(S(x, y), z)$.

Three important continuous t-conorms are:

1. $S_{M}(x, y)=\max (x, y), \quad$ (maximum)

2. $S_{P}(x, y)=x+y-x y, \quad$ (probabilistic sum)
3. $S_{\mathrm{E}}(x, y)=\min (x+y, 1), \quad$ (bounded sum)

Definition 10. Let $\phi$ be an automorphism of the unit interval and $S$ be a t-conorm. Then $S_{\phi}$ denotes the t-conorm that is conjugated to $S$, i.e., $S_{\phi}(x, y)=\phi^{-1}(S(\phi(x), \phi(y)))$, for all $(x, y) \in[0,1]^{2}$.

Definition 11. Let $\phi$ be an automorphism of the unit interval and $I$ be a fuzzy implication. Then $I_{\phi}$ denotes the fuzzy implication that is conjugated to $I$, i.e., $I_{\phi}(x, y)=\phi^{-1}(I(\phi(x), \phi(y)))$, for all $(x, y) \in[0,1]^{2}$.

\section{QL-implications and the first place antitonicity}

First we give two propositions and three lemmas that will play important roles in this section.

Proposition 1. A necessary condition for a $Q L$ implication generated by a t-conorm $S$, a t-norm $T$ and a strong fuzzy negation $n$ to satisfy I1, I4, I5 or I6 is $S(n(x), x)=1$, for all $x \in[0,1]$.

For the case that the t-conorm $S$ is continuous, Proposition 1 can be further expressed by next proposition, according to [4] and [12].

Proposition 2. A necessary condition for a $Q L$ implication generated by a continuous t-conorm $S$, a t-norm $T$ and a strong fuzzy negation $n$ to satisfy I1, I4, I5 or I6 is that there exists an automorphism $\phi$ of the unit interval such that $S=S_{E \phi}$ and $n$ satisfies $n(x) \geq n_{\phi}(x)$, for all $x \in[0,1]$.

Lemma 3. ([10], Theorem 1.13) A fuzzy implication is an S-implication if and only if it satisfies I3, I4 and I6.

Lemma 4. ([10], Theorem 1.14) A fuzzy implication is an R-implication if and only if it satisfies I2, I4 and I5.

Lemma 5. Let $\phi$ be an automorphism of the unit interval. Then a $Q L$-implication $I_{Q L}$ satisfies I1 iff $I_{Q L \phi}$ satisfies $I 1$.

\subsection{Relationship between the first place antitonicity and the other potential properties of QL-implications}

As stated in the Introduction, all QL-implications satisfy I0 and I2. It is also easy to see that each QLimplication satisfies I3. Moreover, a QL-implication generated by a t-conorm $S$, a t-norm $T$ and a strong fuzzy negation is continuous if both $S$ and $T$ are continuous. Thus we will only consider how a QLimplication $I_{Q L}$ satisfies I1, I4, I5 or I6 and the interrelationship between the $I_{Q L}$ 's satisfying them.

Theorem 1. A $Q L$-implication $I_{Q L}$ satisfies I4 iff $I_{Q L}$ is also an $S$-implication. 
Proof. $\Longleftarrow:$ Directly from Lemma 3 .

$\Longrightarrow$ : According to ([12], Remark 2), if $I_{Q L}$ satisfies I4, then $I_{Q L}$ also satisfies I6. Since $I_{Q L}$ always satisfies I3, according to Lemma $3, I_{Q L}$ is also an S-implication.

Remark 1. As stated in the Introduction, an Simplication always satisfies I1. Thus if a QLimplication satisfies I4, then it also satisfies I1.

Next theorem is for the case that the t-conorm $S$ which constructs the QL-implication is continuous, i.e, according to Proposition 2, there exists an automorphism $\phi$ of the unit interval such that $S=S_{\mathrm{E} \phi}$.

Theorem 2. Let $\phi$ be an automorphism of the unit interval. A QL-implication $I_{Q L}$ generated by the $t$ conorm $S_{E \phi}$, a t-norm $T$ and the strong fuzzy negation $n_{\phi}$ satisfies $I_{4}$ iff there exists $s \in[0,+\infty]$ such that $T=T_{\phi}^{s}$, where $T^{s}$ is a Frank t-norm, defined as:

$$
T^{s}(x, y)=\left\{\begin{array}{l}
T_{E}(x, y), \quad s=0 \\
T_{P}(x, y), \quad s=1 \\
T_{M}(x, y), \quad s=+\infty \\
\log _{s}\left(1+\frac{\left(s^{x}-1\right)\left(s^{y}-1\right)}{s-1}\right), \quad \text { otherwise }
\end{array}\right.
$$

Proof. $\Longleftarrow:$ According to the proof of ([12], Corollary $1)$, such a QL-implication $I_{Q L}$ is also an S-implication. Thus according to Lemma $3, I_{Q L}$ satisfies I4.

$\Longrightarrow$ : According to ([12], Remark 2), if $I_{Q L}$ satisfies I4, then $I_{Q L}$ also satisfies I6. Moreover, since $I_{Q L}$ satisfies I2, according to ([4], Lemma 1 (ii)), $I_{Q L}$ satisfies I1. Thus according to ([12], Proposition 9) and ([12], Corollary 1), $T_{\phi^{-1}}$ is a Frank t-norm, i.e., $T=T_{\phi}^{s}$.

A QL-implication satisfies I4 implies that it satisfies I6, but not the reverse. Comparing next theorem and Theorem 2, we can see that there exist QLimplications that satisfy I6 but not I4.

Theorem 3. ([12], Proposition 11) Let $\phi$ be an automorphism of the unit interval. A QL-implication $I_{Q L}$ generated by the $t$-conorm $S_{£ \phi}$, a t-norm $T$ and the strong fuzzy negation $n_{\phi}$ satisfies I6 iff $T_{\phi^{-1}}$ is an ordinal sum of the non-empty family $\left\{\left[a_{m}, b_{m}\right], T_{m}\right\}$, where $T_{m}$ are Frank t-norms defined in (1) with the parameter $s \in[0,+\infty[$.

Remark 2. Since a QL-implication $I_{Q L}$ always satisfies I2, according to ([4], Lemma 1 (ii)), if $I_{Q L}$ satisfies I6, then it also satisfies I1.

Now we consider the conditions under which a QLimplication satisfies I5. Next theorem is for the case that both the t-conorm and the t-norm which construct the QL-implication are continuous.
Theorem 4. Let $\phi$ be an automorphism of the unit interval. A QL-implication $I_{Q L}$ generated by the $t$ conorm $S_{E \phi}$, a continuous t-norm $T$ and a strong fuzzy negation $n$ satisfies I5 iff for all $y \in[0,1]$ :
i) $T(y, y)=n_{\phi}(n(y))$ and
ii) $T(x, y) \geq n_{\phi}(n(x))$, for all $x \in[0, y]$ and
iii) $T(x, y)<n_{\phi}(n(x))$, for all $x \in[y, 1]$.

Proof. $I_{Q L}$ satisfies I5 iff $I_{Q L}(x, y)=\phi^{-1}(\min (\phi(n(x))+\phi(T(x, y)), 1))=1 \Leftrightarrow$ $x \leq y$, which means $\phi(n(x))+\phi(T(x, y)) \geq 1 \Leftrightarrow x \leq y$. Define for all $y \in[0,1], F_{y}(x)=\phi(n(x))+\phi(T(x, y))$, for all $x \in[0,1]$. Then $F_{y}(x) \geq 1$, i.e.,

$T(x, y) \geq n_{\phi}(n(x))$ iff $x \in[0, y]$ and $F_{y}(x)<1$, i.e., $T(x, y)<n_{\phi}(n(x))$ iff $\left.\left.x \in\right] y, 1\right]$. Moreover, since $T$, $n$ and $\phi$ are all continuous, $F_{y}$ is continuous. Thus $F_{y}(y)=1$, i.e., $T(y, y)=n_{\phi}(n(y))$.

Example 1. Let $I_{Q L}$ be the QL-implication defined in Theorem 4 with $T(y, y)=n_{\phi}(n(y))$, for all $y \in[0,1]$. Since $T_{M}$ is the one and only the one t-norm that satisfies $T(y, y)=y$, for all $y \in[0,1]$, we have that if $T=T_{M}$, then $n=n_{\phi}$ and that if $n=n_{\phi}$, then $T=T_{M}$. Actually $I_{Q L}$ generated by $S_{\mathrm{E} \phi}, T_{M}$ and $n_{\phi}$ is an R-implication, i.e., $I_{Q L}(x, y)=\sup \left\{t \in[0,1] \mid T_{\mathrm{E} \phi}(x, t) \leq y\right\}$. According to Lemma $4, I_{Q L}$ satisfies I4 and I5. Moreover, as stated in the Introduction, $I_{Q L}$ satisfies I1.

Remark 3. The QL-implication $I_{Q L}$ defined in Example 1 is also an S-implication, i.e.,

$I_{Q L}(x, y)=S_{\mathrm{E} \phi}\left(n_{\phi}(x), y\right)$. Thus according to Lemma $3, I_{Q L}$ also satisfies I6.

\subsection{QL-implications that satisfy the first place antitonicity}

In this section we will focus on the characterizations of QL-implications satisfying I1. We mainly focus on the continuous cases. We will also indicate whether a QL-implication satisfying I1 is also an S-implication or an R-implication.

Theorem 5. A QL-implication $I_{Q L}$ generated by the t-conorm $S_{E}$, the $t$-norm $T_{M}$ and a strong fuzzy negation $n$ satisfies I1 iff $n(x) \geq n_{0}(x)$, for all $x \in[0,1]$.

Proof. $\Longrightarrow$ : Straightforward from Proposition 2 .

$\Longleftarrow$ : For all $x_{1}, x_{2}$ and $y \in[0,1]$, assume $x_{1}<x_{2}$. If $x_{1} \leq y$, then $I_{Q L}\left(x_{1}, y\right)=S_{\mathrm{Ł}}\left(n\left(x_{1}\right), \min \left(x_{1}, y\right)\right)$ $=S_{\mathrm{E}}\left(n\left(x_{1}\right), x_{1}\right)$. Since $n\left(x_{1}\right) \geq n_{0}\left(x_{1}\right)$,

$I_{Q L}\left(x_{1}, y\right)=1 \geq I_{Q L}\left(x_{2}, y\right)$. Thus we need only consider the situation that $y<x_{1}<x_{2}$. In this case, 
$I_{Q L}\left(x_{1}, y\right)=S_{\mathrm{E}}\left(n\left(x_{1}\right), \min \left(x_{1}, y\right)\right)=S_{\mathrm{E}}\left(n\left(x_{1}\right), y\right)$ and $I_{Q L}\left(x_{2}, y\right)=S_{\mathrm{E}}\left(n\left(x_{2}\right), y\right)$. Since $S_{\mathrm{E}}(\cdot, y)$ is increasing and $n$ is decreasing, we have $I_{Q L}\left(x_{1}, y\right) \geq$ $I_{Q L}\left(x_{2}, y\right)$. Thus for all $x_{1}, x_{2}$ and $y \in[0,1], x_{1}<x_{2}$ implies: $I_{Q L}\left(x_{1}, y\right) \geq I_{Q L}\left(x_{2}, y\right)$, i.e., $I_{Q L}$ satisfies I1.

Corollary 1. Let $\phi$ and $\varphi$ denote two automorphisms of the unit interval. Then a $Q L$-implication $I_{Q L}$ generated by the $t$-conorm $S_{E \phi}$, the $t$-norm $T_{M}$ and a strong fuzzy negation $n_{\varphi}$ satisfies I1 iff $n_{\varphi}(x) \geq n_{\phi}(x)$, for all $x \in[0,1]$.

Proof. $I_{Q L}(x, y)=S_{€ \phi}\left(n_{\varphi}(x), T_{M}(x, y)\right)$

$=\phi^{-1}\left(S_{\mathrm{Ł}}\left(\phi\left(n_{\varphi}(x)\right), T_{M}(\phi(x), \phi(y))\right)\right)$.

Putting $\gamma=\varphi \circ \phi^{-1}$, then $\phi\left(n_{\varphi}(x)\right)=n_{\gamma}(\phi(x))$.

According to Lemma 1 and Lemma $2, \gamma$ is also an automorphism of the unit interval. So $n_{\gamma}$ is a strong fuzzy negation. Thus

$I_{Q L}(x, y)=\phi^{-1}\left(S_{\mathrm{E}}\left(n_{\gamma}(\phi(x)), T_{M}(\phi(x), \phi(y))\right)\right)$

$=\phi^{-1}\left(I_{Q L}^{\prime}(\phi(x), \phi(y))\right)$, where

$I_{Q L}^{\prime}(x, y)=S_{\mathrm{E}}\left(n_{\gamma}(x), T_{M}(x, y)\right)$. According to Theorem $5, I_{Q L}^{\prime}$ satisfies I1 iff $n_{\gamma}(x) \geq n_{0}(x)$, for all $x \in[0,1]$. And according to Lemma $5, I_{Q L}$ satisfies I1 iff $I_{Q L}^{\prime}$ satisfies I1. Thus $I_{Q L}$ satisfies I1 iff $n_{\gamma}(x) \geq n_{0}(x)$, which leads to $n_{\gamma}(\phi(x)) \geq 1-\phi(x)$, which means $\phi\left(n_{\varphi}(x)\right) \geq 1-\phi(x)$, i.e.,

$n_{\varphi}(x) \geq \phi^{-1}(1-\phi(x))=n_{\phi}(x)$, for all $x \in[0,1]$.

Remark 4. According to Example 1 and Remark 3, for the QL-implication $I_{Q L}$ defined in Theorem 5 with $n \geq n_{0}$, if $n=n_{0}$, then $I_{Q L}$ is equivalent to both an S-implication and an R-implication.

On the contrary, we suppose that there exists $\left.x_{0} \in\right] 0,1\left[\right.$ such that $n\left(x_{0}\right)>n_{0}\left(x_{0}\right)$. Then there exists $y_{0}$ such that $x_{0}>y_{0} \geq 1-n\left(x_{0}\right)$, which leads to $n\left(x_{0}\right)+y_{0} \geq 1$, which means $I_{Q L}\left(x_{0}, y_{0}\right)=1$ provided $x_{0}>y_{0}$. Thus $I_{Q L}$ does not satisfy I5. Therefore according to Lemma 4, $I_{Q L}$ is not an R-implication. But $I_{Q L}$ is an Simplication, i.e., $I_{Q L}(x, y)=S_{\mathrm{E}}(n(x), y)$.

Similarly, for the QL-implication $I_{Q L}$ defined in Corollary 1 with $n_{\varphi} \geq n_{\phi}$, if $n_{\varphi}=n_{\phi}$, then $I_{Q L}$ is equivalent to both an S-implication and an Rimplication. If on the contrary $n_{\varphi} \neq n_{\phi}$, then $I_{Q L}$ is not an R-implication but an S-implication, i.e., $I_{Q L}(x, y)=S_{\mathrm{E} \phi}\left(n_{\varphi}(x), y\right)$.

According to Proposition 2, a necessary condition for a QL-implication $I_{Q L}$ generated by a continuous tconorm $S$, a t-norm $T$ and a strong fuzzy negation $n$ to satisfy I1 is that there exists an automorphism $\phi$ of the unit interval such that $S=S_{\mathrm{\ell} \phi}$ and $n \geq n_{\phi}$. The authors of [12] have done the work for the special case that $n=n_{\phi}$. Next theorem gives the sufficient and necessary condition for $I_{Q L}$ of such case to satisfy I1.

Theorem 6. ([12], Proposition 9) Let $\phi$ be an automorphism of the unit interval. A QL-implication $I_{Q L}$ generated by the $t$-conorm $S_{E \phi}$, a t-norm $T$ and the strong fuzzy negation $n_{\phi}$ satisfies $I 1$ iff $T_{\phi^{-1}}$ satisfies the Lipschitz condition, i.e., for all $x_{1}, x_{2}, y \in[0,1]$,

$$
x_{1} \leq x_{2} \Rightarrow T_{\phi^{-1}}\left(x_{2}, y\right)-T_{\phi^{-1}}\left(x_{1}, y\right) \leq x_{2}-x_{1},
$$

There are t-norms sufficient to fulfill the Lipschitz condition (2), here we give examples:

Example 2 According to (1), a Frank t-norm $T^{s}=$ $T_{P}$ if $s=1$ and $T^{s}=T_{\mathrm{E}}$ if $s=0$. It has been stated in ([12], Remark 4) that a t-norm $T$ which is a Frank t-norm or an ordinal sum of the nonempty family $\left\{\left[a_{m}, b_{m}\right], T_{m}\right\}$, where $T_{m}$ are Frank t-norms, always satisfies the Lipschitz condition (2). Thus $T_{P}, T_{\mathrm{E}}$ and $T_{o}$ which is an ordinal sum of the non-empty family $\left\{\left[a_{m}, b_{m}\right], T_{m}\right\}$, where $T_{m}=T_{P}$ or $T_{m}=T_{\mathrm{E}}$ all satisfy the Lipschitz condition (2). Hence according to Theorem 6 , a QL-implication generated by the t-conorm $S_{\mathrm{L} \phi}$, the t-norm $T_{P \phi}, T_{\mathrm{E} \phi}$ or $T_{o \phi}$ and the strong fuzzy negation $n_{\phi}$ satisfies I1.

Remark 5 Let $I_{Q L}$ be a QL-implication generated by the t-conorm $S_{\mathrm{\ell} \phi}$, a t-norm $T$ that $T_{\phi^{-1}}$ satisfies the Lipschitz condition (2) and the strong fuzzy negation $n_{\phi}$. Then according to ([12], Corollary 1), $I_{Q L}$ satisfies I4 iff $T_{\phi^{-1}}$ is a Frank t-norm defined in (1). Thus according to Theorem 1, $I_{Q L}$ is also an S-implication as soon as $T_{\phi^{-1}}$ is a Frank t-norm. Moreover, if $T_{\phi^{-1}}$ is not a Frank tnorm, eg., an ordinal sum of the non-empty family $\left\{\left[a_{m}, b_{m}\right], T_{m}\right\}$, where $T_{m}=T_{P}$ or $T_{m}=T_{\mathrm{E}}$, then $I_{Q L}$ does not satisfy I4. Thus according to Lemma 3 and Lemma 4, it is neither an S-implication nor an R-implication.

Besides the QL-implications generated by the tconorm $S_{\mathrm{E} \phi}$, a t-norm $T$ and the strong fuzzy negation $n_{\phi}$, which we discussed above, there exist other combinations of a t-conorm $S$, a t-norm $T$ and a strong fuzzy negation $n$ to generate a QL-implication $I_{Q L}$ which satisfies I1. It is sufficient but not necessary for $n$ to be $n_{\phi}$ while $S=S_{€ \phi}$. Next we discuss the cases that provided both $S$ and $T$ are continuous, what conditions should $n$ fulfill to make $I_{Q L}$ satisfy I1. Since $T$ is either $T_{M}$, or conjugated to $T_{P}$ or conjugated to $T_{\mathrm{E}}$, or an ordinal sum the non-empty family $\left\{\left[a_{m}, b_{m}\right], T_{m}\right\}$ with $T_{m}$ being conjugated to $T_{P}$ or $T_{\mathrm{E}}$, we have the next theorems and corollaries. First we consider the cases that $T=T_{P}$ or $T$ is conjugated to $T_{P}$. 
Theorem 7. Let $n$ be a strong fuzzy negation and define a mapping $f$ as $f(x)=\frac{1-n(x)}{x}$, for all $x \in] 0,1]$. Then a $Q L$-implication $I_{Q L}$ generated by the $t$-conorm $S_{E}$, the $t$-norm $T_{P}$ and $n$ satisfies $I 1$ iff $f$ is increasing.

Proof. For all $x_{1}, x_{2}$ and $y \in[0,1]$, assume $x_{1}<x_{2}$. $\Longrightarrow$ : In order for $I_{Q L}$ to satisfy I1, it is necessary that $I_{Q L}\left(x_{2}, y\right)=1$ implies $I_{Q L}\left(x_{1}, y\right)=1$, namely, $y \geq \frac{1-n\left(x_{2}\right)}{x_{2}}$ implies $y \geq \frac{1-n\left(x_{1}\right)}{x_{1}}$. Thus

$f\left(x_{2}\right)=\frac{1-n\left(x_{2}\right)}{x_{2}} \geq \frac{1-n\left(x_{1}\right)}{x_{1}}=f\left(x_{1}\right)$, for all $x_{1}<x_{2}$, i.e., $f$ is increasing.

$\Longleftarrow$ : If $I_{Q L}\left(x_{1}, y\right)=1$, then it is always greater than $I_{Q L}\left(x_{2}, y\right)$. If $I_{Q L}\left(x_{2}, y\right)=1$, then since $f$ is increasing, according to the proof above, $I_{Q L}\left(x_{1}, y\right)=1=$ $I_{Q L}\left(x_{2}, y\right)$. Thus we need only consider the situation that $I_{Q L}\left(x_{1}, y\right)=n\left(x_{1}\right)+x_{1} y<1$ and

$I_{Q L}\left(x_{2}, y\right)=n\left(x_{2}\right)+x_{2} y<1$, i.e., $x_{1}>0, x_{2}>0$, $y<\frac{1-n\left(x_{1}\right)}{x_{1}}$ and $y<\frac{1-n\left(x_{2}\right)}{x_{2}}$. Since $f$ is increasing, $\frac{1-n\left(x_{2}\right)}{x_{2}} \geq \frac{1-n\left(x_{1}\right)}{x_{1}}$. Thus:

$n\left(x_{1}\right) x_{1}-n\left(x_{2}\right) x_{1} \geq x_{2}-x_{2} n\left(x_{1}\right)-x_{1}+n\left(x_{1}\right) x_{1}$, which leads to $\frac{n\left(x_{1}\right)-n\left(x_{2}\right)}{x_{2}-x_{1}} \geq \frac{1-n\left(x_{1}\right)}{x_{1}}>y$. Therefore $n\left(x_{1}\right)+x_{1} y>n\left(x_{2}\right)+x_{2} y$, i.e., $I_{Q L}\left(x_{1}, y\right) \geq$ $I_{Q L}\left(x_{2}, y\right)$. Hence $I_{Q L}$ satisfies I1.

Example 3. Let $\phi(x)=x^{2}$. Then $n(x)=\sqrt{1-x^{2}}$ and $f(x)=\frac{1-n(x)}{x}=\frac{1-\sqrt{1-x^{2}}}{x}$, for all $\left.\left.x \in\right] 0,1\right]$. Since $\frac{d f(x)}{d x}=\frac{1-\sqrt{1-x^{2}}}{\sqrt{1-x^{2}} x^{2}} \geq 0, f$ is increasing. Thus $I_{Q L}$ defined by $I_{Q L}(x, y)=S_{€}\left(\sqrt{1-x^{2}}, x y\right)$ satisfies I1.

Corollary 2. Let $\phi, \varphi$ and $\gamma$ be three automorphisms of the unit interval, where $\gamma=\varphi \circ \phi^{-1}$. And define a mapping $f$ as $f(x)=\frac{1-n_{\gamma}(x)}{x}$, for all $\left.\left.x \in\right] 0,1\right]$. Then a QL-implication $I_{Q L}$ generated by the t-conorm $S_{E \phi}$, the $t$-norm $T_{P \phi}$ and $n_{\varphi}$ satisfies I1 iff $f$ is increasing.

Proof. $I_{Q L}(x, y)=S_{\mathrm{E} \phi}\left(n_{\varphi}(x), T_{P \phi}(x, y)\right)$

$=\phi^{-1}\left(S_{\mathrm{E}}\left(\phi\left(n_{\varphi}(x)\right), T_{P}(\phi(x), \phi(y))\right)\right)$.

Since $\gamma=\varphi \circ \phi^{-1}$,

$I_{Q L}(x, y)=\phi^{-1}\left(S_{\mathrm{E}}\left(n_{\gamma}(\phi(x)), T_{P}(\phi(x), \phi(y))\right)\right)$.

According to Lemma 1 and Lemma $2, \gamma$ is also an automorphism of the unit interval. So $n_{\gamma}$ is a strong fuzzy negation. Thus

$I_{Q L}(x, y)=\phi^{-1}\left(I_{Q L}^{\prime}(\phi(x), \phi(y))\right)$, where

$I_{Q L}^{\prime}(x, y)=S_{\mathrm{E}}\left(n_{\gamma}(x), T_{P}(x, y)\right)$. According to Theorem $7, I_{Q L}^{\prime}$ satisfies I1 iff $f$ is increasing. And according to Lemma $5, I_{Q L}$ satisfies I1 iff $I_{Q L}^{\prime}$ satisfies I1. Thus $I_{Q L}$ satisfies I1 iff $f$ is increasing.

Remark 6. Let $I_{Q L}$ be the QL-implication and $f$ be the mapping defined in Theorem 7 with $f$ being increasing. Then $f(x) \leq f(1)=1$, for all $x \in] 0,1]$, which leads to $n(x) \geq n_{0}(x)$, for all $x \in] 0,1]$. Since $n(0)=n_{0}(0)$, we have

$n(x) \geq n_{0}(x)$, for all $x \in[0,1]$. If $n=n_{0}$, then according to Example 2 and Remark $5, I_{Q L}$ is also an S-implication. If on the contrary $n \neq n_{0}$, then consider:

$I_{Q L}\left(x, I_{Q L}(y, z)\right)$

$=\min (n(x)+x \cdot \min (n(y)+y z, 1), 1)$,

which is equivalent to:

i) $n(x)+x(n(y)+y z)$, if $n(y)+y z<1$ and $n(x)+x(n(y)+y z)<1$

ii) 1 otherwise,

and $I_{Q L}\left(y, I_{Q L}(x, z)\right)$

$=\min (n(y)+y \cdot \min (n(x)+x z, 1), 1)$,

which is equivalent to:

i) $n(y)+y(n(x)+x z)$, if $n(x)+x z<1$ and $n(y)+y(n(x)+x z)<1$

ii) 1 otherwise.

Since $n$ is continuous and since $g(x)=\frac{1-x}{n(x)}$, $x \in[0,1[$ cannot be constant, there exist $x_{0}, y_{0} \in\left[0,1\left[\right.\right.$ such that $x_{0} \neq y_{0}$ and $g\left(x_{0}\right) \neq g\left(y_{0}\right)$. Let $g\left(x_{0}\right)>g\left(y_{0}\right)$. Then we have $\frac{\frac{1-n\left(y_{0}\right)}{y_{0}}-n\left(x_{0}\right)}{x_{0}}<\frac{\frac{1-n\left(x_{0}\right)}{x_{0}}-n\left(y_{0}\right)}{y_{0}}$. Thus there exists $z_{0}$ such that

$\frac{\frac{1-n\left(y_{0}\right)}{y_{0}}-n\left(x_{0}\right)}{x_{0}} \leq z_{0}<\frac{\frac{1-n\left(x_{0}\right)}{x_{0}}-n\left(y_{0}\right)}{y_{0}} \leq \frac{1-n\left(y_{0}\right)}{y_{0}}$.

Therefore $x_{0}, y_{0}, z_{0}$ satisfy $n\left(y_{0}\right)+y_{0} z_{0}<1$ and $n\left(x_{0}\right)+x_{0}\left(n\left(y_{0}\right)+y_{0} z_{0}\right)<1$ and

$n\left(y_{0}\right)+y_{0}\left(n\left(x_{0}\right)+x_{0} z_{0}\right) \geq 1$, which means $I_{Q L}\left(y_{0}, I_{Q L}\left(x_{0}, z_{0}\right)\right)=1$ while $I_{Q L}\left(x_{0}, I_{Q L}\left(y_{0}, z_{0}\right)\right)<1$. Thus $I_{Q L}$ does not satisfy I4. According to Lemma 3 and Lemma $4, I_{Q L}$ is neither an S-implication nor an R-implication.

Similarly, let $I_{Q L}$ be the QL-implication and $f$ be the mapping defined in Corollary 2 with $f$ being increasing, we have $n_{\varphi}(x) \geq n_{\phi}(x)$, for all $x \in[0,1]$. If $n_{\varphi}=n_{\phi}$, then $I_{Q L}$ is also an S-implication. If on the contrary $n_{\varphi} \neq n_{\phi}$, then $I_{Q L}$ is neither an S-implication nor an R-implication.

Theorem 8. A QL-implication $I_{Q L}$ generated by the t-conorm $S_{E}$, the $t$-norm $T_{E}$ and a strong fuzzy negation $n$ satisfies I1 iff $n=n_{0}$.

Proof. $\Longleftarrow:$ Straightforward from Theorem 6 and Example 2 .

$\Longrightarrow$ : Take $0<x_{1}<x_{2}<1$. Since $1-x_{2}<1-x_{1}<$ $2-n\left(x_{1}\right)-x_{1}$, for all $\left.x_{1} \in\right] 0,1\left[\right.$, there exists $y_{0}$ such that $1-x_{1}<y_{0}<2-n\left(x_{1}\right)-x_{1}$ and $1-x_{2}<y_{0}$. Thus $I_{Q L}\left(x_{1}, y_{0}\right)=n\left(x_{1}\right)+x_{1}+y_{0}-1<1$ and $I_{Q L}\left(x_{2}, y_{0}\right)=\min \left(n\left(x_{2}\right)+x_{2}+y_{0}-1,1\right)$. If $I_{Q L}$ satisfies I1, then it is necessary that $I_{Q L}\left(x_{2}, y_{0}\right)<1$, i.e., 
$n\left(x_{2}\right)+x_{2}+y_{0}-1<1$. Namely, $y_{0}<2-n\left(x_{1}\right)-x_{1}$ implies $y_{0}<2-n\left(x_{2}\right)-x_{2}$. Thus define $f$ as $f(x)=n(x)+x, f$ must be decreasing for all $x \in$ ]0,1[. Since $n$ is continuous, $f$ is continuous. Thus $f$ must be decreasing for all $x \in[0,1]$. Moreover, we have $f(0)=f(1)=1$. Therefore $f(x)=1$, for all $x \in[0,1]$, which means $n(x)=1-x$, for all $x \in[0,1]$, i.e., $n=n_{0}$.

Corollary 3. Let $\phi$ and $\varphi$ be two automorphisms of the unit interval. Then a QL-implication $I_{Q L}$ generated by the $t$-conorm $S_{E \phi}$, the t-norm $T_{E \phi}$ and a strong fuzzy negation $n_{\varphi}$ satisfies I1 iff $n_{\varphi}=n_{\phi}$.

Proof. $I_{Q L}(x, y)=S_{\mathrm{Ł} \phi}\left(n_{\varphi}(x), T_{\mathrm{\complement} \phi}(x, y)\right)$

$=\phi^{-1}\left(S_{\mathrm{E}}\left(\phi\left(n_{\varphi}(x)\right), T_{\mathrm{E}}(\phi(x), \phi(y))\right)\right)$.

Putting $\gamma=\varphi \circ \phi^{-1}$, then $\phi\left(n_{\varphi}(x)\right)=n_{\gamma}(\phi(x))$.

According to Lemma 1 and Lemma $2, \gamma$ is also an automorphism of the unit interval. So $n_{\gamma}$ is a strong fuzzy negation. Thus $I_{Q L}(x, y)$

$=\phi^{-1}\left(S_{€}\left(n_{\gamma}(\phi(x)), T_{€}(\phi(x), \phi(y))\right)\right)$

$=\phi^{-1}\left(I_{Q L}^{\prime}(\phi(x), \phi(y))\right)$,

where $I_{Q L}^{\prime}(x, y)=S_{€}\left(n_{\gamma}(x), T_{€}(x, y)\right)$.

According to Theorem $8, I_{Q L}^{\prime}$ satisfies I1 iff

$n_{\gamma}=n_{0}$. And according to Lemma $5, I_{Q L}$ satisfies I1 iff $I_{Q L}^{\prime}$ satisfies I1. Thus $I_{Q L}$ satisfies I1 iff $n_{\gamma}=n_{0}$. So $n_{\gamma}(x)=1-x, n_{\gamma}(\phi(x))=1-\phi(x)$, being equivalent to $\phi\left(n_{\varphi}(x)\right)=1-\phi(x)$, which means

$n_{\varphi}(x)=\phi^{-1}(1-\phi(x))$, for all $x \in[0,1]$. Hence

$n_{\varphi}=n_{\phi}$.

Remark 7. According to Example 2 and Remark 5, the QL-implication defined in Theorem 8 with $n=n_{0}$ and the QL-implication defined in Corollary 3 with $n_{\varphi}=n_{\phi}$ are equivalent to $\mathrm{S}$ implications.

Next we consider the t-norm $T$ which constructs the QL-implication to be an ordinal sum of the non-empty family $\left\{\left[a_{m}, b_{m}\right], T_{m}\right\}$, where $T_{m}=T_{P}$ or $T_{m}=T_{\mathrm{E}}$. Because of space limitation, we omit the proofs of the theorems and corollaries below.

Theorem 9. Let $\left\{\left[a_{m}, b_{m}\right]\right\}$ be a non-empty family of non-overlapping, closed, proper subintervals of $[0,1]$ and $T_{o}$ be an ordinal sum of $\left\{\left[a_{m}, b_{m}\right], T_{m}\right\}$, where $T_{m}=T_{P}$. Moreover, let $n$ be a strong fuzzy negation and define $f_{m}(x)=\frac{1-n(x)-a_{m}}{x-a_{m}}$, for all $m$ and $x \in$ ]$\left.a_{m}, b_{m}\right]$. Then a $Q L$-implication $I_{Q L}$ generated by the $t$-conorm $S_{E}, T_{o}$ and $n$ satisfies $I 1$ iff $n(x) \geq n_{0}(x)$, for all $x \in[0,1]$ and $f_{m}$ is increasing, for all $m$.

Theorem 10. Let $\left\{\left[a_{m}, b_{m}\right]\right\}$ be a non-empty family of non-overlapping, closed, proper subintervals of $[0,1]$ and $T_{o}$ be an ordinal sum of $\left\{\left[a_{m}, b_{m}\right], T_{m}\right\}$, where $T_{m}=T_{E}$. Then a QL-implication $I_{Q L}$ generated by the t-conorm $S_{E}, T_{o}$ and a strong fuzzy negation $n$ satisfies I1 iff

i) $n(x)=n_{0}(x)$, if $x \in\left[a_{m}, b_{m}\right]$ and

ii) $n(x) \geq n_{0}(x)$, otherwise.

Synthesize Theorem 9 and Theorem 10, we have the next corollary.

Corollary 4. Let $\left\{\left[a_{i}, b_{i}\right]\right\}$ and $\left\{\left[a_{j}, b_{j}\right]\right\}$ be two non-empty families of non-overlapping, closed, proper subintervals of $[0,1]$ and $\left\{\left[a_{m}, b_{m}\right]\right\}=\left\{\left[a_{i}, b_{i}\right]\right\} \cup$ $\left\{\left[a_{j}, b_{j}\right]\right\} . T_{o}$ is an ordinal sum of $\left\{\left[a_{m}, b_{m}\right], T_{m}\right\}$, which is defined as:

$$
\begin{cases}a_{i}+\left(b_{i}-a_{i}\right) T_{P}\left(\frac{x-a_{i}}{b_{i}-a_{i}}, \frac{y-a_{i}}{b_{i}-a_{i}}\right), & (x, y) \in\left[a_{i}, b_{i}\right]^{2} \\ a_{j}+\left(b_{j}-a_{j}\right) T_{E}\left(\frac{x-a_{j}}{b_{j}-a_{j}}, \frac{y-a_{j}}{b_{j}-a_{j}}\right), & (x, y) \in\left[a_{j}, b_{j}\right]^{2} \\ T_{M}(x, y), & \text { otherwise }\end{cases}
$$

Moreover, let $n$ be a strong fuzzy negation and define $f_{i}(x)=\frac{1-n(x)-a_{i}}{x-a_{i}}$, for all $i$ and $\left.\left.x \in\right] a_{i}, b_{i}\right]$. Then $a$ $Q L$-implication $I_{Q L}$ generated by the t-conorm $S_{E}, T_{o}$ and $n$ satisfies I1 iff

i) $f_{i}$ is increasing, for all $i$, and

ii) $n(x)=n_{0}(x)$, for all $x \in\left[a_{j}, b_{j}\right]$, and

iii) $n(x) \geq n_{0}(x)$, for all $x \notin\left[a_{i}, b_{i}\right]$ and all $x \notin\left[a_{j}, b_{j}\right]$.

For the QL-implication being conjugated to the one defined in Corollary 4, we have the next corollary.

Corollary 5. Let $T_{o}$ be a t-norm defined by (3), $n$ be a strong fuzzy negation and $\phi$ be an automorphism of the unit interval. Define $f_{i}(x)=\frac{1-\phi\left(n\left(\phi^{-1}(x)\right)\right)-a_{i}}{x-a_{i}}$, for all $i$ and $\left.x \in] a_{i}, b_{i}\right]$. Then a $Q L$-implication $I_{Q L}$ generated by the $t$-conorm $S_{E \phi}, T_{o \phi}$ and $n$ satisfies $I 1$ iff

i) $f_{i}$ is increasing, for all $i$, and

ii) $n(x)=n_{\phi}(x)$, for all $x \in\left[a_{j}, b_{j}\right]$, and

iii) $n(x) \geq n_{\phi}(x)$, for all $x \notin\left[a_{i}, b_{i}\right]$ and all $x \notin\left[a_{j}, b_{j}\right]$.

Proof. Suppose $n^{\prime}(x)=\phi\left(n\left(\phi^{-1}(x)\right)\right)$. According to Lemma $5, I_{Q L}$ satisfies I1 iff $I_{Q L \phi^{-1}}$, which is expressed as $I_{Q L \phi^{-1}}(x, y)=S_{\mathrm{E}}\left(n^{\prime}(x), T_{o}(x, y)\right)$, satisfies I1. According to Corollary $5, I_{Q L \phi^{-1}}$ satisfies I1 iff

i) $f_{i}^{\prime}$ is increasing, for all $i$, and

ii) $n^{\prime}(x)=n_{0}(x)$, for all $x \in\left[a_{j}, b_{j}\right]$, and 
iii) $n^{\prime}(x) \geq n_{0}(x)$, for all $x \notin\left[a_{i}, b_{i}\right]$ and all $x \notin\left[a_{j}, b_{j}\right]$,

where $f_{i}^{\prime}(x)=\frac{1-n^{\prime}(x)-a_{i}}{x-a_{i}}$, for all $\left.\left.x \in\right] a_{i}, b_{i}\right]$. The three conditions are equivalent to

i) $f_{i}$ is increasing, for all $i$, and

ii) $n(x)=n_{\phi}(x)$, for all $x \in\left[a_{j}, b_{j}\right]$, and

iii) $n(x) \geq n_{\phi}(x)$, for all $x \notin\left[a_{i}, b_{i}\right]$ and all $x \notin\left[a_{j}, b_{j}\right]$.

\section{Conclusions}

In this paper, we have studied the QL-implications generated by a t-conorm, a t-norm and a strong fuzzy negation that satisfy the properties which are required to obtain a suitable conclusion in fuzzy inference. Especially the first place antitonicity (property I1 in the Introduction) of QL-implications has been studied. Theorem 1 to Theorem 4 state the general relationship between a QL-implication satisfying the first place antitonicity and the other properties. Moreover, Theorem 5 to Theorem 10 together with Corollary 1 to Corollary 5 state sufficient and necessary conditions for the QL-implications generated by different combinations of a t-conorm, a t-norm and a strong fuzzy negation to satisfy the first place antitonicity. Whether the QL-implications which satisfy the first place antitonicity are equivalent to S- or R- implications have been illustrated in Remarks 4 to 7 .

\section{References}

[1] M. Baczynski J. Drewniak, Conjugacy classes of fuzzy implication, in: B. Reusch (Eds.), Computational intelligence: Theory and Applications. Springer-Verlag, Berlin (Lecture Notes in Computer Science, Vol. 1625), 1999, pp. 287-298.

[2] M. Baczynski, Residual implications revisited. Notes on the Smets-Magrez Theorem, Fuzzy Sets and Systems 145 (2004) 267-277.

[3] M. Baczynski, J. Balasubramaniam, On the characterizations of $(S, N)$-implications, Fuzzy Sets and Systems, to appear.

[4] H. Bustince, P. Burillo, F. Soria, Automorphisms, negations and implication operators, Fuzzy Sets and Systems 134 (2003) 209-229.
[5] D. Dubois, H Prade, Fuzzy sets in approximate reasoning, part 1: inference with possibility distributions, Fuzzy Sets and Systems 40 (1991) 143202.

[6] D. Dubois, H Prade, Fuzzy sets in approximate reasoning, part 2: logical approaches, Fuzzy Sets and Systems 40 (1991) 202-244.

[7] D. Dubois, H Prade, Gradual inference rules in approximate reasoning, Information Sciences 61 (1992) 103-122.

[8] J.C. Fodor, On fuzzy implication operators, Fuzzy Sets and Systems 42 (1991) 293-300.

[9] J.C. Fodor, Contrapositive symmetry of fuzzy implications, Fuzzy Sets and Systems 69 (1995) 141156.

[10] J.C. Fodor, M. Roubens, Fuzzy Preference Modelling and Multicriteria Decision Support, Kluwer Academic Publishers, Netherlands, 1994.

[11] G.J. Klir, B. Yuan, Fuzzy Sets and Fuzzy Logic, Theory and Applications, Prentice Hall, USA, 1995.

[12] M. Mas, M. Monserrat, J. Torrens, QLimplications versus D-implications, Kybernetika 42 (2006) 351-366.

[13] D. Ruan, E.E. Kerre, Fuzzy implication operators and generalized fuzzy method of cases, Fuzzy Sets and Systems 54 (1993) 23-37.

[14] D. Ruan, E.E. Kerre, Fuzzy IF-THEN Rules in Computational Intelligence: Theory and Applications, Kluwer Academic Publishers, Boston, 2000.

[15] P. Smets, P. Magrez, Implication in fuzzy logic, Internat. J. Approx. Reason. 1 (1987) 327-347.

[16] E. Trillas, C.D. Campo, S. Cubillo, When QMoperators are implication functions and conditional fuzzy relations, International Journal of Intelligent Systems 15 (2000) 647-655.

[17] R.R. Yager, On global requirements for implication operators in fuzzy modus ponens, Fuzzy Sets and Systems 106 (1999) 3-10. 